\title{
Mechanical thrombectomy of acute basilar artery occlusion: single center experience
}

\begin{abstract}
Purpose: Acute basilar artery occlusion (BAO) is a devastating neurological condition associated with a poor clinical outcome and a high mortality rate. Mechanical thrombectomy using a retrievable stent applied shortly after symptom onset could increase a good functional outcome, improving survival rate in patients with acute BAO. First clinical trials using stent retrievers have shown promising high recanalization rates. This study aimed to evaluate the feasibility, safety and efficacy of mechanical thrombectomy.
\end{abstract}

Material and Methods:Seven consecutive patients ( 2 female, 5 male) with a mean age of 59,4 (range 40-82) with acute BAO undergone to endovascular therapy: all patients were treated with mechanical thrombectomy using a retrievable stent (in 5 patients with Solitaire-Covidien system, in 2 patients Revive-Codman system); two of these received in addition a intra-arterial thrombolysis (IAT), one was additionally treated with intra-venous thrombolysis (IVT). Successful recanalization was defined as Thrombolysis in Cerebral Infarction (TICI) grade $2 \mathrm{~b}$ or 3 . Good outcome was defined as modified Rankin Scale (mRS) score of $0-2$ at 3 months.

Results: Median NIHSS score at onset was 15,8 (range 12-22). Median procedure time to maximal recanalization was 72 minutes (range 35-135). Recanalization was achieved in $100 \%(7 / 7)$ of patients. One symptomatic parenchymal hemorrhage occurred in a patient treated additionally with intra-arterial thrombolysis. Median NIHSS score at 7 days from treatment was 7,2 (range 0-20). At 3 months a good outcome (mRS 0-2) was observed in $71,4 \%(5 / 7)$; overall mortality at 3 months was $28,6 \%(2 / 7)$.

Conclusion: Mechanical thrombectomy in BAO presents high recanalization rate, with a very low complication rate, improving good outcome and survival rates in patients with BAO.

Keywords: Mechanical Thrombectomy; Acute Basilar Artery Occlusion; Neurological condition; Endovascular therapy; Intra-venous thrombolysis; Recanalization; Atherothrombotic occlusions; Acute ischemic stroke
Volume 5 Issue I - 2016

\author{
Andrea Giorgianni,', Giuseppe Craparo, ${ }^{2}$ \\ Carlo Pellegrino,' Irene Chiara De Bernardi,' \\ Federico Carimati, ${ }^{3}$ Maria Luisa De \\ Lodovici, ${ }^{3}$ Alberto Terrana,' Camilla Micieli, I \\ Serena Monaco, ${ }^{4}$ Cristina Gallo, ${ }^{2}$ Fabio \\ Baruzzi' \\ 'Department of Neuroradiology, Ospedale di Circolo, Italy \\ ${ }^{2}$ Department of Neuroradiology, Ospedale Civico di Palermo, \\ Italy \\ ${ }^{3}$ Department of Neurology, Ospedale di Circolo, Italy \\ ${ }^{4}$ Department of Neurology, Ospedale Civico di Palermo, Italy
}

Correspondence: Dr.Alberto Terrana, M.D., University of Insubria, Circolo Varese Hospital,V.le Borri 53, Italy, Tel +39-3284250630 Gmail albertoterrana@libero.it

Received:June 17,2016 | Published: July II, 2016

\section{Introduction}

Acute basilar artery occlusion (BAO) is associated with a very poor outcome and has the highest mortality rate among intracranial large-vessel occlusions. ${ }^{1,2}$ The main causes of acute BAOs are a atherothrombotic occlusion, due to local thrombosis on a stenosis, and embolic occlusions, due to cardiac or arterio-arterial thromboembolism. Atherothrombotic occlusions account for 26$36 \%$ and embolic occlusions are founded in a $30-35 \%$ of cases of acute BAO. ${ }^{2,3}$ For a good functional outcome in BAO, recanalization is the most important prognostic factor. ${ }^{2-5}$ Recanalization rates are approximately with a intravenous and intra-arterial pharmacology thrombolysis respectively of $53 \%$ and of $65 \%$, with rates of death or dependency of $78 \%$ and $76 \%{ }^{6}$ Recently, mechanical thrombectomy using self-expanding retrievable stent have initiated a new era in endovascular stroke therapy, allowing a fast and efficient flow restoration. Controlled trials and different personal series showed that stent retrievers can achieve a very high rate of recanalization of up to $90 \%$, improving clinical outcome in patients with acute intracranial large-vessel occlusion. ${ }^{7-12}$ Despite these findings, in BAO clinical data and detailed analyses of mechanical thrombectomy with stent retrievers are still sparse.

\section{Materials and Methods}

\section{Patients}

Since February 2012, we prospectively collected data of patients with acute ischemic stroke treated with endovascular treatment. From this prospectively collected cohort, we retrospectively analyzed all patients with acute ischemic stroke with BAO undergoing to mechanical thrombectomy between February 2012 and February 2015. Seven patients ( 2 female, 5 male) with a median age of 59,4 (range 40-82) with BAO were treated. On admission, all patients were evaluated by a stroke neurologist and assessed by means of the NIHSS. (Table $1 \&$ 2) All patients underwent an initial imaging protocol that included non-enhanced CT scan and an Angio-CT. The inclusion criteria for endovascular treatment were baseline NIHSS score $>9$; no intracerebral hemorrhage detected on the cranial CT, BAO detectetion on conventional angiography correlated with neurological deficit.

\section{Endovascular Treatment}

Endovascular treatments were performed within 8 hours of stroke onset (mean time 4 hours, range 2-6) by an Interventional neuroradiologist. Under local anesthesia using a transfemoral approach, a 6 French guide catheter was placed in the proximal vertebral artery. To prevent the occurrence of a thromboembolic event during the procedure, a solution of 2000 international units heparin and $0.9 \%$ normal saline $(1000 \mathrm{~mL})$ were administered in continuous through the guiding catheter. A cerebral diagnostic angiography was performed to assess vessel occlusion and collateral flow to the affected vessel territory. After basilar arterial occlusion was demonstrated, diagnostic catheter was exchanged with a $6 \mathrm{~F}$ guide catheter (Neuron, Penumbra, California, USA). A microcatheter on a microwire 0.018- 
inch (Rebar 18, Covidien, California, USA) was introduced into the target vessel. After that, a retrievable stent (in 5 cases Solitaire, Covidien, California, USA; in other 2 cases Revive, Codman, California, USA) was introduced through microcatheter and deployed across the occluded segment. (Figure $1 \& 2$ ) After the stent was maintained in place for least 3 minutes, microcatheter and stent were gently pulled back together and withdrawn outside the body through the guide catheter. A control angiogram was performed to assess status of recanalization and possible distal embolic events. If recanalization was unsuccessful, the procedure was repeated, with a maximum of 4 was allowed. In addition to mechanical thrombectomy two patients received an intra-arterial thrombolysis (IAT) with recombinant tissue-plasminogen activator (rt-PA) $(60 \mathrm{ml}$ and $15 \mathrm{ml}$ respectively of Alteplase, ActyliseO) and one patient received intra-venous rt-PA thrombolysis $(72 \mathrm{ml} \mathrm{-} \mathrm{0,9ml/kg} \mathrm{-} \mathrm{of} \mathrm{Alteplase,} \mathrm{ActyliseOे)} \mathrm{(Table} 1 \&$ 2). Recanalization was classified according to the Thrombolysis in Cerebral Infarction (TICI) grading scale. ${ }^{13}$ TICI grades 3 and $2 b$ were rated as sufficient recanalization and TICI grades $2 \mathrm{a}$ to 0 were rated as insufficient. In two cases it was reported a slowdown of circle in the right posterior cerebral artery treated by an intravenous injection of $500 \mathrm{mg}$ of acid acetylsalicylic (FlectadolÒ). All patients after procedure were observed for at least 24 hours in an intensive care unit and CT was performed. The NIHSS score was assessed at day 7 by a stroke neurologist. Clinical outcome was assessed at 3 months by a stroke neurologist according to the modified Rankin Scale (mRS) score. ${ }^{14}$
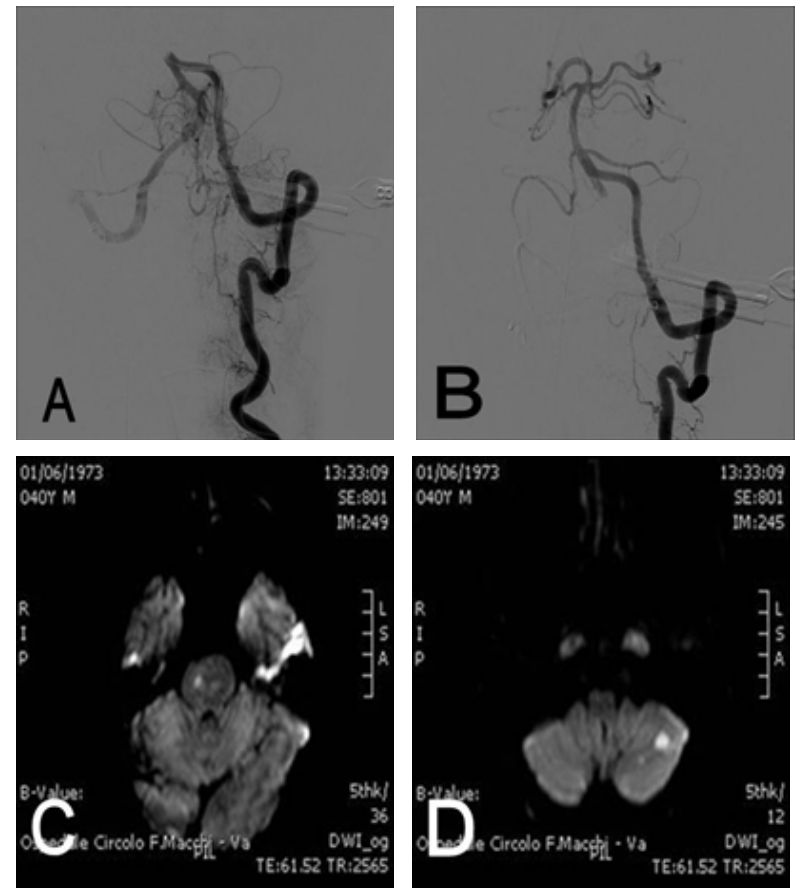

Figure I Digital subtraction angiography of basilar artery occlusion before (A) and after (B) treatment with mechanical thrombectomy. MRI sequences in DWI show a pontal (C) and cerebellar (D) ischemia.

\section{Results}

Patients' characteristics and clinical result are presented in Table 1 $\& 2$. The median NIHSS score on admission was 18,7 (range 16-22). The occlusion site was the proximal to distal basilar artery segments in 1 patient, the mid-basilar and distal artery in 4 patients, and the distal basilar artery in 1 patient. Overall, an acceptable recanalization grade (TICI 3 or $2 b$ ) was achieved in all patients. The median procedure time to recanalization was 72 minute (range 35-135). A symptomatic parenchymal hemorrhage occurred in one patient who received intraarterial urokinase infusion additionally (Patient $n^{\circ} 3$, Table $1 \& 2$ ). Median NIHSS score after 7 days was 7,2 (range 0-20) with a mean gain of 8,6 NIHSS score. At 3 months a good outcome (mRS $0-2$ ) was observed in $71,4 \%(5 / 7)$; overall mortality at 3 months was $28,6 \%$ $(2 / 7)$.

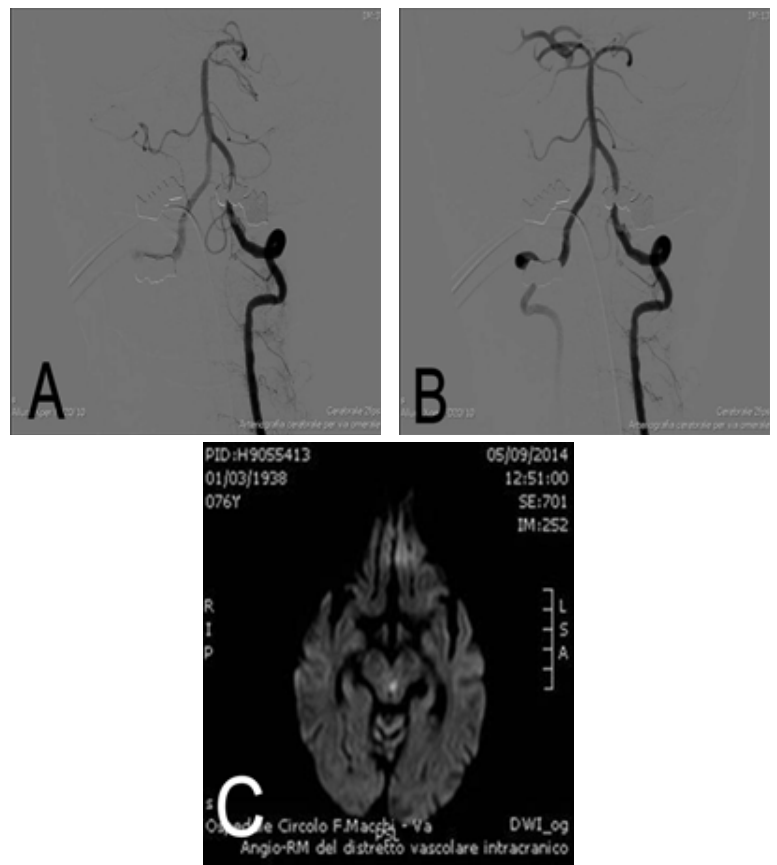

Figure 2 Digital subtraction angiography of midbasilar-distal artery occlusion before (A) and after (B) treatment with mechanical thrombectomy. MRI sequences in DWI (C) shows a mesenchefalic paramedian-left ischemia.

\section{Discussion}

The use of retrievable stents for the treatment of endovascular ischemic stroke increased recanalization rates up to $80 \%-94 \%{ }^{11,15-18}$ On the other hand, there is still a diminished literature with only few studies on mechanical thrombectomy with stent retrievers in patients with acute BAO. ${ }^{7,18}$ In Baek et al. ${ }^{8}$ study, 25 patients with BAO were treated within 8 hours of stroke onset with mechanical thrombectomy using Solitaire stent, reported a $88 \%$ rate of survival at 3 months $(22 / 25)$, and more than half of the survivors $(55 \%, 12 / 22)$ had a good outcome. Costalat et al.. ${ }^{11}$ who included sixteen patients with acute BAO in a larger series of fifty patients with large vessel occlusion, achieved a recanalization $(\mathrm{TICI}=3)$ in $81 \%(13 / 16)$ with a good outcome $(\mathrm{mRS}$ $0-2)$ rate at 3 months of $44 \%(7 / 16)$ and a mortality rate of $25 \%(4 / 16)$. Mordasini et al. ${ }^{7}$ in a series of 14 patients treated with a stent retriever thrombectomy, obtained a $28.6 \%(4 / 14)$ of good outcome (mRS 0-2), and a mortality rate of $35.7 \%(5 / 14){ }^{7}$ According to Mordasini et al. ${ }^{7}$ Mourand et al. ${ }^{19}$ obtained a good outcome in $35 \%$ (11/31), with a mortality rate of $32 \%(10 / 31) .{ }^{19}$ Espinosa de Rueda et al. ${ }^{20}$ reported, in a series of 18 patients with acute BAO who were directly treated with mechanical thrombectomy with either the Solitaire stent (10/18) or the Trevo stent (8/10), a good outcome was recorded in 50\% (9/18), and the mortality rate was $22.3 \%$ at 3 months. Wang et al. ${ }^{21}$ in his personal series of 18 patients with BAO all treated with mechanical thrombectomy (10 patients only with mechanical thrombectomy, 8 patients treated in addition with intra-arterial thrombolysis) reported a recanalization rate of $94.4 \%$ with a mortality rate, according to the literature, of $27.8 \%$. The literature shows a contrast between a high recanalization rate and clinically good outcome rate. ${ }^{7,8,11,19-21}$ These findings might be attributable to the small personal series, but also to 
different factors indicated as predictors of outcome as initial NIHSS score, age, time from onset to treatment, thrombus localization. ${ }^{2,3,22-24}$

Mechanical thrombectomy with a retrievable stent is a relative safe technique with a very low rate of compliances, in particular hemorrhage, as we can see in the literature: Mordasini et al. ${ }^{7}$ reported no device-related complications or symptomatic hemorrhage. Mourand et al. ${ }^{19}$ reported a symptomatic hemorrhage rate of $16 \%$ and Espinosa de Rueda et al. ${ }^{20}$ reported no symptomatic hemorrhage in their personal cases. ${ }^{19,20}$ According to literature our study confirms the safety of mechanical thrombectomy with retrievable stent in patients with BAO. In our study just one parenchymal hemorrhage occurred in a patient treated by a multimodal approach with retrievable stent and intra-arterial thrombolysis. Parenchymal hemorrhage may be caused by the intra-arterial pharmacology treatment respect to mechanical treatment by stent. The limitations of our study included the small number of patients and the lack of a control group.

\section{Conclusion}

Our preliminary study suggests that an endovascular approach using retrievable stents in acute BAO presents a high recanalization rate with a very low complication rates and a good outcome rate. Mechanical treatment by using a retrievable stent can significantly reduce the mortality rate in this subset of patients with acute stroke, who otherwise have an extremely poor prognosis. Mechanical thrombectomy represents treatment of choice for acute basilar artery occlusion.

\section{Acknowledgments}

None.

\section{Conflicts of interest}

None.

\section{References}

1. Hacke W, Zeumer H, Ferbert A, Intra-arterial thrombolytic therapy improves outcome in patients with acute vertebrobasilar occlusive disease. Stroke. 1988:19(10):1216-1222.

2. Schonewille WJ, Wijman CA, Michel Pet al. Treatment and outcomes of acute basilar artery occlusion in the basilar artery international cooperation study (BASICS): a prospective registry study. Lancet Neurol. 2009:8(8):724-730.

3. Arnold M, Nedeltchev K, Schroth G et al. Clinical and radiological predictors of recanalisation and outcome of 40 patients with acute basilar artery occlusion treated with intra-arterial thrombolysis. $J$ Neurol Neurosurg Psychiatry. 2004:75(6):857-862.

4. Schulte-Altedorneburg G, Hamann GF, Mull Met al. Outcome of acute vertebrobasilar occlusions treated with intra-arterial fibrinolysis in 180 patients. AJNR Am J Neuroradiol. 2006:27(10):2042-2047.

5. Davis SM, Donnan GA Basilar artery thrombosis: recanalization is the key. Stroke. 2006:37(9):2440.

6. Lindsberg PJ, Mattle HP Therapy of basilar artery occlusion: a systematic analysis comparing intra-arterial and intravenous thrombolysis. Stroke. 2006:37(3):922-928.

7. Mordasini P, Brekenfeld C, Byrne JV et al. Technical feasibility and application of mechanical thrombectomy with the Solitaire FR revascularization device in acute basilar artery occlusion. AJNR Am J Neuroradiol. 2013:34(1):159-163.
8. Baek JM, Yoon SK, Jung MSet al. Acute basilar artery occlusion: outcome of mechanical thrombectomy with Solitaire stent within 8 hours of stroke onset. AJNR Am J Neuroradiol. 2014:35(5):989-993.

9. Saver JL, Jahan R, Levy EI et al. Solitaire flow restoration device versus the Merci retriever in patients with acute ischaemic stroke (SWIFT): a randomized, parallel-group, non-inferiority trial. Lancet. 2012:380(9649):1241-1249.

10. Nogueiro RG, Lutsep HL, Jovin TG et al. Trevo versus Merci retrievers for thrombectomy revascularization of large vessel occlusions in acute ischemic stroke (TREVO 2): a randomized trial. Lancet. 2012:380(9849):1231-1240.

11. Costalat V, Machi P, Lobotesis K et al. Rescue, combined, and standalone thrombectomy in the management of large vessel occlusion stroke using the Solitaire device: a prospective 50-patient singlecenter study: timing, safety, and efficacy. Stroke. 2011:42(7):1929-1935.

12. Cohen JE, Leker RR, Moscovici S et al. Stent-based mechanical thrombectomy in acute basilar artery occlusion. J Clin Neurosci. 2011:18(12):1718-1720.

13. Higashida RT, Furlan AJ, Roberts $\mathrm{H}$ et al. Trial design and reporting standards for intra-arterial cerebral thrombolysis for acute ischemic stroke. Stroke. 2003:34(8):e109-e137.

14. van Swieten JC, Koudstaal PJ, Visser $\mathrm{MC}$ et al. Interobserver agreement for the assessment of handicap in stroke patients. Stroke. 1988:19(5):604-607.

15. Castaño C, Dorado L, Guerrero C et al. Mechanical thrombectomy with the Solitaire AB device in large artery occlusions of the anterior circulation: a pilot study. Stroke. 2010:41(8):1836-1840.

16. Roth C, Papanagiotou P, Behnke S et al. Stent-assisted mechanical recanalization for treatment of acute intracerebral artery occlusions. Stroke. 2010:41(11):2559-2567.

17. Park H, Hwang GJ, Jin SC et al. Aretrieval thrombectomy technique with the Solitaire stent in a large cerebral artery occlusion. Acta Neurochir. 2011:153(8):1625-1631.

18. Fesl G, Holtmannspoetter M, Patzing M et al. Mechanical Thrombectomy in Basilar Artery Thrombosis: Technical Advances and Safety in a 10Year Experience. Cardiovasc Intervent Radiol. 2014:37(2):355-361.

19. Mourand I, Machi P, Milhaud D et al. Mechanical thrombectomy with the Solitaire device in acute basilar artery occlusion. $J$ Neurointervent Surg. 2014:6(3):200-204.

20. Espinosa de Rueda M, Parrilla G, Zamarro J et al. Treatment of acute vertebrobasilar occlusion using thrombectomy with stent retrievers: initial experience with 18 patients. AJNR Am J Neuroradiol. 2013:34(5):1044-1048.

21. Wang L, Shi W, Su Z et al. Endovascular treatment of severe acute basilar artery occlusion. J Clinic Neuroscience. 2015:22(1):195-198.

22. Brandt T, von Kummer R, Muller-Kuppers M et al. Thrombolytic therapy of acute basilar artery occlusion. Variables affecting recanalization and outcome. Stroke. 1996:27(5):875-881.

23. Jung S, Mono ML, Fischer U et al. (2011) Three-month and long-term outcomes and their predictors in acute basilar artery occlusion treated with intra-arterial thrombolysis. Stroke. 1996:42(7):1946-1951.

24. Strbian D, Sairanen T, Silvennoinen H et al. Thrombolysis of basilar artery occlusion: impact of baseline ischemia and time. Ann Neurol. 2013:73(6):688-694. 